TRANSACTIONS OF THE

AMERICAN MATHEMATICAL SOCIETY

Volume 354, Number 1, Pages 75-89

S 0002-9947(01)02855-0

Article electronically published on August 21, 2001

\title{
LOCALISATION HOMOTOPIQUE ET TOUR DE TAYLOR POUR UNE CATÉGORIE ABÉLIENNE
}

\author{
OLIVIER RENAUDIN
}

\begin{abstract}
RÉSUMÉ. On indique comment une sous-catégorie colocalisante d'une catégorie abélienne induit une localisation dans la catégorie dérivé. Ceci permet une nouvelle construction de la tour de Taylor d'un foncteur à valeur dans une catégorie de module.
\end{abstract}

La localisation d'une catégorie abélienne par rapport à une sous-catégorie a été étudiée par Gabriel G]. On étudie ici la localisation à la Bousfield [B1, B2] des catégories dérivées des catégories abéliennes. Il s'agit de remplacer, au mieux, un complexe de chaînes par un autre dont l'homologie appartient à une sous-catégorie prescrite.

Il apparaît que les colocalisations considérées par Gabriel induisent une localisation au niveau homotopique, ce qui résout le problème sous des hypothèses naturelles. Ces localisations s'appliquent par exemple aux catégories de foncteurs, vis-à-vis des sous-catégories de foncteurs polynômiaux de degré prescrit; on produit ainsi des tours de Taylor similaires à celles de Johnson et McCarthy JMcC1, JMcC2].

La section 1 montre comment obtenir la localisation par rapport à une souscatégorie colocalisante. On se donne une catégorie abélienne (localement petite) $\mathcal{A}$, possédant assez de projectifs, et une sous-catégorie colocalisante $\mathcal{B}$ telle que la catégorie quotient $\mathcal{A} / \mathcal{B}$ possède assez de projectifs. On note $C h(\mathcal{A})$ la catégorie des complexes de chaînes connectifs sur $\mathcal{A}$ et $\operatorname{Ho}(C h(\mathcal{A}))$ la catégorie homotopique associée. On considère la classe $h \mathcal{B}$ des morphismes de $H o(C h(\mathcal{A}))$ qui induisent un isomorphisme via les foncteurs $\left[-, \Sigma^{q} B\right]$, pour $q \in \mathbb{N}$ et pour tout objet $B$ de $\mathcal{B}$. Une $h \mathcal{B}$-localisation de $X$ est alors un morphisme terminal parmi les morphismes de $h \mathcal{B}$ de source $X$. Un objet $L$ est dit $h \mathcal{B}$-local si le foncteur $[-, L]$ transforme les morphismes de $h \mathcal{B}$ en isomorphismes (ces notions sont reprises dans l'appendice). On vérifie (théorème 1.2) :

Théorème 0.1. Sous les hypothèses précédentes, tout objet de $H o(C h(\mathcal{A}))$ possède une $h \mathcal{B}$-localisation, et un objet de $\operatorname{Ho}(C h(\mathcal{A}))$ est $h \mathcal{B}$-local si et seulement si son homologie est dans $\mathcal{B}$.

Remarquons qu'il s'agit de localisations relativement à des familles de foncteurs "cohomologiques" : $X \mapsto\left[X, \Sigma^{*} B\right]$. La contravariance de ces foncteurs ne semble pas permettre d'utiliser les arguments usuels de colimite [B1].

La section 2 indique comment de telles localisations s'appliquent à des catégories de foncteurs. L'utilisation de localisation dans ce cadre est due à Jeff Smith (cours

Received by the editors November 14, 2000.

2000 Mathematics Subject Classification. Primary 18E35, 18 G55.

(C)2001 American Mathematical Society 
à Orsay, 1992, [FS]), et a inspiré ce travail. Dans cette section, $\mathcal{A}$ sera une catégorie de foncteurs de source une petite catégorie possédant sommes finies et objet nul et de but une catégorie de module. La sous-catégorie colocalisante $\mathcal{B}$ sera la souscatégorie des foncteurs de degré inférieur ou égal à $n$. Les hypothèses du théorème 0.1 sont vérifiées, et on obtient donc un foncteur localisation qui associe, à un complexe de foncteurs, le plus "proche" complexe de foncteurs dont l'homologie soit de degré inférieur ou égal à $n$. La filtration polynômiale de la catégorie de foncteurs induit ainsi dans la catégorie homotopique une tour de localisations, comparable à la tour de Taylor construite par Johnson et McCarthy [JMcC1, JMcC2].

Dans la section 3, on vérifie, dans les conditions de la première section mais avec quelques hypothèses supplémentaires, l'existence d'une structure de catégorie de modèle associée à la localisation (théorème 3.1 ) :

Théorème 0.2. Si la catégorie $\mathcal{A}$ est une catégorie de Grothendieck possédant un ensemble de petits générateurs projectifs, alors la catégorie $C h(\mathcal{A})$ possède une structure de catégorie de modèle fermée avec la classe hB comme classe d'équivalences faibles.

Cette section n'est pas utilisée dans le reste de l'article, mais on espère que le cas simple traité ici puisse éclairer la même structure dans des cadres plus complexes (comme par exemple [MV]).

Enfin, un appendice rappelle des définitions et résultats utilisés dans l'article, concernant les notions de localisation, de sous-catégorie colocalisante et de catégorie de modèle fermée.

Remerciements. Merci vivement à Vincent Franjou pour, entre autre, l'aide considérable qu'il a prêté à la rédaction de cet article. Merci également à Jeff Smith pour ses explications et au rapporteur pour ses suggestions.

\section{Localisation}

1.1. Complexes de chaînes et catégorie de modèle fermée. Etant donnée une catégorie abélienne $\mathcal{A}, C h(\mathcal{A})$ désigne la catégorie des complexes de chaînes concentrés en degrés positifs (i.e. supérieurs ou égaux à 0 ) sur $\mathcal{A}$. Le prolongement d'un foncteur $F: \mathcal{C} \rightarrow \mathcal{D}$ est le foncteur $C h(\mathcal{C}) \rightarrow C h(\mathcal{D})$ obtenu en appliquant $F$ en chaque degré. Il sera également noté $F$ dans la suite.

Soient $\mathcal{A}$ une catégorie abélienne localement petite et $\mathcal{B}$ une sous-catégorie colocalisante de $\mathcal{A}$ :

$$
\mathcal{B} \hookrightarrow \mathcal{A} \stackrel{T}{\longrightarrow} \mathcal{A} / \mathcal{B}
$$

On note $S$ l'adjoint à gauche du foncteur quotient $T$ : pour tout objet $X \operatorname{de} \mathcal{A} / \mathcal{B}$, et pour tout $Y$ de $\mathcal{A}$, il existe un isomorphisme naturel:

$$
\operatorname{Hom}_{\mathcal{A}}(S(X), Y) \simeq \operatorname{Hom}_{\mathcal{A} / \mathcal{B}}(X, T(Y)) .
$$

L'unité d'adjonction : $I d_{\mathcal{A} / \mathcal{B}} \rightarrow T \circ S$ est alors un isomorphisme [G, p.371].

Cette situation se prolonge aux complexes :

$$
C h(\mathcal{B}) \hookrightarrow C h(\mathcal{A}) \stackrel{T}{\longrightarrow} C h(\mathcal{A} / \mathcal{B}) .
$$

On note $H_{n}(A)$ le $n$-ième "groupe" d'homologie du complexe $A$.

Le fait que la catégorie $\mathcal{A}$ ait assez de projectifs permet de munir $C h(\mathcal{A})$ d'une structure de catégorie de modèle fermée [Q, Ch.II,4]. La définition d'une catégorie de modèle fermée est rappelée en appendice. 
Les équivalences faibles sont les quasi-isomorphismes : les morphismes induisant un isomorphisme en homologie. Les cofibrations sont les injections de conoyau projectif en chaque degré. Les fibrations peuvent être caractérisées comme les morphismes qui sont des surjections en degrés strictement positifs.

Ceci assure l'existence de $H o(C h(\mathcal{A}))$, la catégorie homotopique associée à $C h(\mathcal{A})$. L'ensemble des morphismes $X \rightarrow Y$ de $H o(C h(\mathcal{A}))$ est désigné par $[X, Y]$. Le foncteur suspension est noté $\Sigma$, son adjoint à droite $\Omega$, et on notera éventuellement, pour $n<0: \Sigma^{n}=\Omega^{-n}$.

Le diagramme de $H o(C h(\mathcal{A})): X \rightarrow Y \rightarrow Z$, est appelé suite de cofibration Q, Ch.I,3] s'il est isomorphe à un diagramme $A \rightarrow B \rightarrow C$ de $C h(\mathcal{A})$, où $A \rightarrow B$ est une cofibration entre cofibrants, de cofibre $C$, c'est-à-dire une suite exacte de cofibrants. Toute suite exacte de $C h(\mathcal{A})$ est faiblement équivalente à une suite exacte de cofibrants.

1.2. Construction de la localisation. Le but de cette section est de vérifier l'existence de localisation et acyclisation homotopique associées à une sous-catégorie colocalisante $\mathcal{B}$ d'une catégorie abélienne localement petite $\mathcal{A}$ possédant assez de projectifs. Dans toute la suite, on fait l'hypothèse suivante :

(H) $\mathcal{A} / \mathcal{B}$ possède assez de projectifs.

Elle assure l'existence de la structure de catégorie de modèle fermée $\operatorname{sur} C h(\mathcal{A} / \mathcal{B})$, et en particulier l'existence de $H o(C h(\mathcal{A} / \mathcal{B}))$.

Cette hypothèse est vérifiée pour toute sous-catégorie colocalisante si $\mathcal{A}$ possède des revêtements projectifs [P, p.182]. Elle l'est également dans le cadre des catégories de foncteurs considérées dans la section 2.

Proposition 1.1. La paire de foncteurs adjoints $(S, T)$ induit une paire d'adjoints :

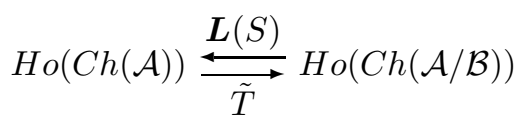

telle que l'unité de l'adjonction $: I d \rightarrow(\tilde{T} \circ \boldsymbol{L}(S))$ soit un isomorphisme.

Démonstration. L'exactitude de $T$ implique que son prolongement préserve les équivalences faibles et les fibrations. La paire $(S, T)$ est donc une paire de Quillen [DS p.114] [DHK p.13], ce qui assure l'existence de l'adjonction $(\boldsymbol{L}(S), \tilde{T})$.

Pour que l'unité de l'adjonction soit un isomorphisme, il suffit [Q, p.4.7] que l'adjointe d'une équivalence faible $S(X) \stackrel{f}{\rightarrow} Y, X \in C h(\mathcal{A} / \mathcal{B}), Y \in C h(\mathcal{A})$, soit une équivalence faible $X \stackrel{\breve{f}}{\rightarrow} T(Y)$. Or, le foncteur $T$ préserve les équivalences faibles, et on a : $\check{f}=T(f) \circ u$ où $u$ est l'isomorphisme unité $: I d \rightarrow(T \circ S)$.

Définition. On appelle $h \mathcal{B}$-équivalence un morphisme $f$ de $C h(\mathcal{A})$ tel que, pour tout $B \in \mathcal{B},\left[f, \Sigma^{*} B\right]$ soit un isomorphisme. La classe des $h \mathcal{B}$-équivalences est notée $h \mathcal{B}$. Un objet $A$ de $C h(\mathcal{A})$ est $h \mathcal{B}$-acyclique si $\left[A, \Sigma^{*} B\right]=0$ pour tout $B \in \mathcal{B}$.

Le théorème suivant donne l'existence de la $h \mathcal{B}$-acyclisation et de la $h \mathcal{B}$-localisation, ainsi qu'une caractérisation des objets $h \mathcal{B}$-locaux.

Théorème 1.2. Soit $\mathcal{A}$ une catégorie abélienne localement petite possédant assez de projectifs. Soit $\mathcal{B}$ une sous-catégorie colocalisante de $\mathcal{A}$ telle que la catégorie quotient $\mathcal{A} / \mathcal{B}$ possède assez de projectifs.

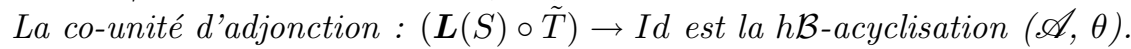


Il existe une $h \mathcal{B}$-localisation $(\mathscr{L}, \eta)$, et on a, pour tout $X \in C h(\mathcal{A})$, une suite de cofibration : $\mathscr{A}(X) \rightarrow X \rightarrow \mathscr{L}(X)$.

De plus, $L$ est $h \mathcal{B}$-local si et seulement si $H_{*}(L) \in \mathcal{B}$.

Ceci est démontré à la suite de la proposition 1.3

Définition. On appelle $\pi \mathcal{B}$-équivalence un morphisme $f$ de $C h(\mathcal{A})$ tel que $H_{*}(f)$ soit un $\mathcal{B}$-isomorphisme. La classe des $\pi \mathcal{B}$-équivalences est notée $\pi \mathcal{B}$.

Proposition 1.3. La co-unité d'adjonction : $(\boldsymbol{L}(S) \circ \tilde{T}) \rightarrow I d$ constitue une colo-

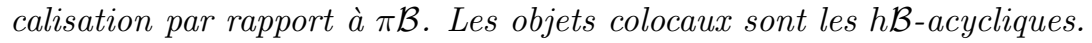

Démonstration. L'unité de l'adjonction $(\boldsymbol{L}(S), \tilde{T})$ étant un isomorphisme (proposition 1.1) la co-unité $(\boldsymbol{L}(S) \circ \tilde{T}) \rightarrow I d$ est une comonade idempotente.

Elle définit une colocalisation, par rapport aux morphismes de $\mathrm{Ho}(C h(\mathcal{A}))$ transformés en isomorphisme par $\boldsymbol{L}(S) \circ \tilde{T}$, donc ceux transformés en isomorphisme par $\tilde{T}$, car $\tilde{T} \circ \boldsymbol{L}(S) \simeq I d$. Il s'agit donc bien d'une colocalisation par rapport à $\pi \mathcal{B}$ car $H_{*} \circ \tilde{T} \simeq T \circ H_{*}$.

Si $A$ est colocal, $A \simeq(\boldsymbol{L}(S) \circ \tilde{T})(A)$, et donc :

$$
\left[A, \Sigma^{*} B\right] \simeq\left[\boldsymbol{L}(S) \circ \tilde{T}(A), \Sigma^{*} B\right] \simeq\left[\tilde{T}(A), \Sigma^{*} T(B)\right]=0
$$

soit $A$ hB-acyclique.

Réciproquement, soit $A$ un $h \mathcal{B}$-acyclique. Par définition, $A$ est colocal si et seulement si, pour tout $f \in \pi \mathcal{B},[A, f]$ est un isomorphisme. Il suffit alors de considérer le cas où $f \in \pi \mathcal{B}$ est une cofibration de $C h(\mathcal{A})$. Sa cofibre $C_{f}$ vérifie alors : $H_{*}\left(C_{f}\right) \in \mathcal{B}$. Comme $A$ est $h \mathcal{B}$-acycliques, en particulier pour tout $n, k \in \mathbb{N}$, $\left[A, \Sigma^{n} H_{k}\left(C_{f}\right)\right]=0$. Notons $P_{k}$ le k-ième étage de la tour de Postnikov de $C_{f}$. Par récurrence, la suite exacte longue associée à la suite de cofibration: $\Sigma^{k} H_{k}\left(C_{f}\right) \rightarrow$ $P_{k} \rightarrow P_{k-1}$ montre, pour tout $k \in \mathbb{N},\left[A, \Sigma^{n} P_{k}\right]=0$. Comme $C_{f} \simeq \operatorname{Lim}_{k} P_{k}$, une suite exacte courte de Milnor:

$$
0 \rightarrow \operatorname{Lim}_{k}^{1}\left[A, \Sigma^{n-1} P_{k}\right] \rightarrow\left[A, \Sigma^{n} C_{f}\right] \rightarrow \operatorname{Lim}_{k}\left[A, \Sigma^{n} P_{k}\right] \rightarrow 0
$$

montre que pour tout $n \in \mathbb{Z}:\left[A, \Sigma^{n} C_{f}\right]=0$. La suite exacte longue associée à la suite exacte courte $: 0 \rightarrow X \stackrel{f}{\rightarrow} Y \rightarrow C_{f} \rightarrow 0$ montre enfin que $[A, f]$ est un isomorphisme.

Démonstration du théorème 1.2. D'après la proposition $1.3,(\boldsymbol{L}(S) \circ \tilde{T}) \rightarrow I d$ est la $\pi \mathcal{B}$-colocalisation, et vérifie donc, pour tout $X$ de $H o(C h(\mathcal{A}))$, la propriété universelle : $(\boldsymbol{L}(S) \circ \tilde{T})(X) \rightarrow X$ est terminal parmi les morphismes de source $\pi \mathcal{B}$ colocal et de but $X$. En utilisant l'équivalence entre $\pi \mathcal{B}$-colocaux et $h \mathcal{B}$-acycliques, celle-ci devient : $(\boldsymbol{L}(S) \circ \tilde{T})(X) \rightarrow X$ est terminal parmi les morphismes de source $h \mathcal{B}$-acyclique et de but $X$, ce qui démontre la première assertion.

On suit la méthode de B3] pour construire la localisation. Soit $L$ la cofibre homotopique de $(\boldsymbol{L}(S) \circ \tilde{T})(X) \rightarrow X$. La suite exacte longue induite par la suite de cofibration :

$$
(\boldsymbol{L}(S) \circ \tilde{T})(X) \rightarrow X \rightarrow L
$$

montre que $X \rightarrow L$ est $h \mathcal{B}$-localisante.

Une suite de cofibration peut être représentée par une suite exacte courte. Par la suite exacte longue d'homologie et la proposition [1.3. on a : $H_{*}(L) \in \mathcal{B}$. Soit $f \in h \mathcal{B}$. Par définition, pour tout $B \in \mathcal{B}$, pour tout $n \in \mathbb{N},\left[f, \Sigma^{n} B\right]$ est un 
isomorphisme. Donc, par les tours de Postnikov, $[f, L]$ est un isomorphisme pour tout $L \in H o(C h(\mathcal{A}))$ vérifiant $H_{*}(L) \in \mathcal{B}$. Ceci implique que $L$ est $h \mathcal{B}$-local. Le morphisme $X \rightarrow L$ est donc bien une $h \mathcal{B}$-localisation. Ceci permet de définir la paire $(\mathscr{L}, \eta)$.

On vient de voir que si $H_{*}(L) \in \mathcal{B}$, alors $L$ est $h \mathcal{B}$-local. Réciproquement, le foncteur $h \mathcal{B}$-localisation $\mathscr{L}$ ainsi construit prenant comme valeurs les objets $A$ tel que $H_{*}(A) \in \mathcal{B}$, les objets $h \mathcal{B}$-locaux vérifient cette propriété.

Remarque. Les considérations ci-dessus sont également valables dans la catégorie des complexes de chaînes bornés inférieurement.

\section{TOURS DE TAYLOR}

Soient $A$ un anneau, et $\mathcal{E}$ une petite catégorie possédant sommes finies et objet nul. Nous considérons ici la catégorie $\mathcal{F}$ des foncteurs de la catégorie $\mathcal{E}$ dans la catégorie des $A$-modules.

Nous allons appliquer des localisations par rapport à la sous-catégorie des foncteurs additifs, et, plus généralement, par rapport aux catégories $\mathcal{F}_{n}$ des foncteurs polynômiaux de degré $\leq n$. Elles forment une suite croissante de sous-catégories :

$$
\mathcal{F}_{0} \subset \mathcal{F}_{1} \subset \ldots \subset \mathcal{F}_{n} \subset \mathcal{F}_{n+1} \subset \ldots \subset \mathcal{F} .
$$

auxquelles on va appliquer notre théorème de localisation homotopique. On obtient, pour tout $F \in H o(C h(\mathcal{F}))$, une tour de localisations :

$$
F \longrightarrow \cdots \longrightarrow \mathscr{L}_{n+1}(F) \longrightarrow \mathscr{L}_{n}(F) \longrightarrow \cdots \longrightarrow \mathscr{L}_{1}(F) \longrightarrow \mathscr{L}_{0}(F)=F(0)
$$

où $\mathscr{L}_{n}$ désigne le foncteur $h \mathcal{F}_{n}$-localisation de $\operatorname{Ho}(C h(\mathcal{F}))$. Une telle localisation approxime un foncteur par un complexe de chaînes de foncteurs, dont l'homologie est polynômiale de degré $\leq n$. La valeur de cette homologie est intéressante. Par exemple, si $F \in \mathcal{F}$, avec $F(0)=0$, les foncteurs $H_{k}\left(\mathscr{L}_{1}(F)\right)$ ne sont autre que les foncteurs dérivés stable de $F$ étudiés par Dold et Puppe dans DP].

Précisons un peu. La catégorie $\mathcal{F}$ est une catégorie de Grothendieck où les foncteurs $P_{E}=A[\operatorname{Hom}(E,-)], E \in \mathcal{E}$, forment un ensemble de petits générateurs projectifs.

Soit $\mathcal{F}^{\prime(n)}$ la catégorie des foncteurs, à $n$ variables, nuls dès qu'une variable est nulle. La restriction du foncteur diagonale:

$$
\Delta_{n}^{*}: \mathcal{F}^{(n)} \longrightarrow \mathcal{F}
$$

possède un adjoint à droite :

$$
c r_{n}: \mathcal{F} \longrightarrow \mathcal{F}^{(n)},
$$

appelé $n$-ième déviation (ou cross-effect).

Les foncteurs $c r_{n}$ mesurent le défaut d'additivité des foncteurs de $\mathcal{F}$. Le concept de cross-effect a été introduit par Eilenberg et Mac Lane [EMcL section 9] On trouvera dans [JMcC2, section 1] une construction par récurrence des foncteurs déviations et divers résultats, dont l'adjonction $\left(\Delta_{n}^{*}, c r_{n}\right)$ [JMcC2, p.8]. La construction par récurrence montre que les foncteurs déviations sont exacts, et préservent donc l'homologie.

Un foncteur $F \in \mathcal{F}$ est dit polynômial de degré inférieur ou égal à $n$ si $c r_{n+1}(F)=$ 0 , et on note $\mathcal{F}_{n}$ la sous-catégorie de $\mathcal{F}$ formée de ces foncteurs.

Définition. Un foncteur est dit $n$-diagonalisable s'il appartient à $\Delta_{n}^{*}\left(\mathcal{F}^{\prime(n)}\right)$. 
La proposition suivante est essentiellement le résultat de Pirashvili [Pi].

Proposition 2.1. Soient $F$ et $G$ deux objets de $C h(\mathcal{F})$. Si l'homologie de $F$ est de degré inférieur ou égal à $n-1$ et si $G$ est $n$-diagonalisable, alors : $[G, F] \simeq 0$. Tout complexe de foncteurs $n$-diagonalisable en chaque degré est $h \mathcal{F}_{n-1}$-acyclique.

Démonstration. Les foncteurs $\Delta_{n}^{*}$ et $c r_{n}$ sont exacts, si bien que l'adjonction donne un isomorphisme :

$$
\left[\Delta_{n}^{*}(G), F\right] \simeq\left[G, c r_{n}(F)\right] .
$$

Ceci démontre la première partie de la proposition, ainsi que la deuxième dans le cas des suspensions itérées d'un foncteur discret. Le cas général s'en déduit alors par récurrence sur le squelette.

Une réciproque est montrée au théorème 2.4 .

Corollaire 2.2. Tout foncteur $(n+1)$-diagonalisable est $\mathcal{F}_{n}$-colocal.

On note $: c r_{n}^{\delta}=\Delta_{n}^{*} \circ c r_{n}$ et $c$ la co-unité de l'adjonction $: c r_{n}^{\delta}(F) \longrightarrow F$. Pour tout $F$ dans $\mathcal{F}$, on a une suite exacte naturelle en $F$ :

$$
c r_{n}^{\delta}(F) \stackrel{c}{\longrightarrow} F \longrightarrow m_{n-1}(F) \longrightarrow 0 .
$$

Proposition 2.3. Pour chaque entier positif n, la sous-catégorie $\mathcal{F}_{n}$ est colocalisante et la catégorie $\mathcal{F} / \mathcal{F}_{n}$ possède assez de projectifs.

Démonstration. Pour montrer que $\mathcal{F}_{n-1}$ est colocalisante, on constate d'abord qu'elle est épaisse de par l'exactitude du foncteur $c r_{n}$, et on utilise ensuite la caractérisation donnée par la proposition duale de la proposition A.1 [G, p.372]. Pour tout $F \in \mathcal{F}, m_{n-1}(F)$ est le plus grand quotient de $F$ dans $\mathcal{F}_{n-1}$. En effet, notant $u$ l'unité de l'adjonction $\left(\Delta_{n}^{*}, c r_{n}\right)$, la composée :

$$
\left(c r_{n}\left(c_{F}\right) \circ u_{c r_{n}(F)}\right): c r_{n}(F) \rightarrow\left(c r_{n} \circ c r_{n}^{\delta}\right)(F) \rightarrow c r_{n}(F)
$$

est l'identité de $c r_{n}(F)$. Cela montre, par l'exactitude de $c r_{n}$, que $c r_{n}\left(m_{n-1}(F)\right)=$ 0 , soit $m_{n-1}(F) \in \mathcal{F}_{n-1}$.

Soit $Q$ un quotient de $F$, de degré inférieur ou égal à $n-1$. La composée: $c r_{n}^{\delta}(F) \stackrel{c}{\longrightarrow} F \longrightarrow Q$ est nulle car $c r_{n}^{\delta}(F)$ est $\mathcal{F}_{n-1}$-colocal, si bien que $F \longrightarrow Q$ se factorise par $m_{n-1}(F)$. Le quotient $m_{n-1}(F)$ est donc maximal parmi les quotients de $F$ appartenant à $\mathcal{F}_{n-1}$.

Enfin, la suite exacte :

$$
\operatorname{cr}_{n}^{\delta}(F) \stackrel{c}{\longrightarrow} F \longrightarrow m_{n-1}(F) \longrightarrow 0
$$

montre que si $m_{n-1}(F)$ est nul, $F$ est quotient d'un colocal. La catégorie $\mathcal{F}_{n-1}$ est donc bien colocalisante. On note $S_{n-1}$ l'adjoint à gauche du foncteur passage au quotient $T_{n-1}$ :

$$
\mathcal{F}_{n-1} \stackrel{m_{n-1}}{\longleftrightarrow} \mathcal{F} \frac{S_{n-1}}{T_{n-1}} \mathcal{F} / \mathcal{F}_{n-1}
$$

Comme la catégorie $\mathcal{F}$ possède assez de projectifs, il existe pour tout $F \in \mathcal{F}$ un projectif $P \in \mathcal{F}$ et un épimorphisme $P \rightarrow F$. La composée : $\operatorname{cr}_{n}^{\delta}(P) \stackrel{c}{\rightarrow} P \rightarrow$ $F$ induit un épimorphisme par $T_{n-1}: \mathcal{F} \longrightarrow \mathcal{F} / \mathcal{F}_{n-1}$. Il reste à vérifier que 
$T_{n-1}\left(c r_{n}^{\delta}(P)\right)$ est projectif dans $\mathcal{F} / \mathcal{F}_{n-1}$. L'adjonction $\left(S_{n-1}, T_{n-1}\right)$ et le fait que $\operatorname{cr}_{n}^{\delta}(P)$ est $\mathcal{F}_{n-1}$-colocal entrainent les isomorphismes :

$$
\begin{aligned}
\operatorname{Hom}\left(T_{n-1}\left(c r_{n}^{\delta}(P)\right),-\right) \circ T_{n-1} & \simeq \operatorname{Hom}\left(\left(S_{n-1} \circ T_{n-1}\right)\left(c r_{n}^{\delta}(P)\right),-\right) \\
& \simeq \operatorname{Hom}\left(c_{n}^{\delta}(P),-\right) .
\end{aligned}
$$

Ceci assure que $\operatorname{Hom}\left(T_{n-1}\left(c r_{n}^{\delta}(P)\right),-\right)$ est exact.

La proposition précédente montre que les théorèmes 1.2 et 3.1 s'appliquent à la situation colocalisante $\mathcal{F}_{n} \hookrightarrow \mathcal{F}$. Le théorème suivant donne une caractérisation des $h \mathcal{F}_{n}$-acycliques. Sa démonstration utilise des notations et un résultat de la section 3.

Théorème 2.4. Dans $H o(C h(\mathcal{F}))$, un objet est $h \mathcal{F}_{n}$-acyclique si et seulement s'il est isomorphe à un objet $(n+1)$-diagonalisable en chaque degré, qui peut être choisi cofibrant.

Démonstration. Par la proposition 3.3, un $h \mathcal{F}_{n}$-acyclique est isomorphe à un objet $V$-libre (somme de $V$ en chaque degré), où $V=S_{n}\left(U^{\prime}\right), U^{\prime}$ un générateur projectif de $\mathcal{F} / \mathcal{F}_{n}$. Il suffit de vérifier que l'on peut choisir $V(n+1)$-diagonalisable et projectif.

D'après la démonstration de la proposition 2.3. $U^{\prime}$ peut être choisi de la forme : $T_{n}\left(c r_{n+1}^{\delta}(P)\right)$, avec $P$ projectif, et alors $V=\left(S_{n} \circ T_{n}\right)\left(c r_{n+1}^{\delta}(P)\right) \simeq c r_{n+1}^{\delta}(P)$ convient.

Des tours comparables ont été obtenues par B. Johnson et R. McCarthy [JMcC1, JMcC2], dans un cadre similaire, et par B. Richter [R] dans le cadre des $\Gamma$-groupes abéliens. Bien que moins abstraite, la méthode de [JMcC2] utilise également l'adjonction $\left(\Delta_{n+1}^{*}, c r_{n+1}\right)$ et plus particulièrement le cotriple $\Delta_{n+1}^{*} \circ c r_{n+1}$. Ils forment, pour chaque foncteur $F \in C h(\mathcal{F})$, la cocomplétion relative à ce cotriple, c'est a dire le complexe total de la résolution associée, et obtiennent ainsi l'acyclisation. La cofibre homotopique, notée $P_{n} F$, constitue le n-ième cran de leur tour de Taylor, et vérifie [JMcC1 Theorem 3.11] la propriété [JMcC2, Lemma 2.11] :

La flèche $F \rightarrow P_{n} F$ est universelle à quasi-isomorphisme près relativement aux transformations naturelles vers un foncteur dont l'homologie est de degré $n$. Autrement dit, la flèche $F \rightarrow P_{n} F$ induit la $h \mathcal{F}_{n}$-localisation dans $H o(C h(\mathcal{F}))$.

\section{CAtÉGORIE DE MOdèLE}

Il s'agit d'obtenir une structure de catégorie de modèle fermée avec $h \mathcal{B}$ comme classe d'équivalences faibles. L'essentiel de la section est consacré à la construction d'une factorisation. Ceci sera effectué sous les hypothèses détaillées dans le théorème 3.1

3.1. Structure de catégorie de modèle localisée. On munit $C h(\mathcal{A})$ de la structure localisée de Bousfield relative à $h \mathcal{B}$ :

Définitions. Soit $f$ un morphisme $\operatorname{de} C h(\mathcal{A})$ :

- $f$ est une $h \mathcal{B}$-cofibration si $f$ est une cofibration.

- $f$ est une $h \mathcal{B}$-équivalence faible si $f \in h \mathcal{B}$, c'est-à-dire : pour tout $B \in \mathcal{B},\left[f, \Sigma^{*} B\right]$ est un isomorphisme.

- $f$ est une $h \mathcal{B}$-fibration si et seulement si $f$ a la propriété de relèvement à droite par rapport à toute cofibration appartenant à $h \mathcal{B}$. 
Les cofibrants sont donc encore les objets projectifs en chaque degré.

Théorème 3.1. Soit $C h(\mathcal{A})$ une catégorie de Grothendieck possédant un ensemble de petits générateurs projectifs. Soit $\mathcal{B}$ une sous-catégorie colocalisante de $\mathcal{A}$ telle que la catégorie quotient $\mathcal{A} / \mathcal{B}$ possède assez de projectifs. La catégorie $C h(\mathcal{A})$ et les classes de $h \mathcal{B}$-équivalences faibles, $h \mathcal{B}$-cofibrations, et $h \mathcal{B}$-fibrations définies cidessus constituent une catégorie de modèle fermé.

Démonstration. L'existence de la factorisation MC5(ii) (axiome de la liste de [DS] rappelé à la section $\mathrm{A.3}$ ) est assurée par le théorème 3.4 section 3.3 et le reste est standard B1, B2].

3.2. Remarques préliminaires. Cette section contient quelques remarques et notations, ainsi qu'une caractérisation des $h \mathcal{B}$-acycliques donnée par la proposition 3.3, qui seront utilisées dans la section suivante.

On note $\left\{U_{i}\right\}_{i \in I}$ l'ensemble de petits générateurs projectifs de $\mathcal{A}, U_{i}$ petit signifiant que $\operatorname{Hom}\left(U_{i},-\right)$ commute avec toute colimite. En particulier, toute flèche de $U_{i}$ dans une somme se factorise par une sous-somme finie. On note $U=\bigoplus_{I} U_{i}$; c'est un générateur projectif de $\mathcal{A}$.

Les foncteurs : $\mathrm{E}_{i}=\left(\operatorname{Hom}\left(U_{i},-\right) \circ H_{*}\right): C h(\mathcal{A}) \rightarrow$ Ab commutent donc aux colimites, et procurent une caractérisation des équivalences faibles de $C h(\mathcal{A})$ :

$$
\bigcap_{I} \mathrm{E}_{i}^{-1}(\{\text { isomorphismes }\})=\{\text { équivalences faibles }\} \text {. }
$$

Définition. Soit $A$ un objet de $\mathcal{A}$. Un projectif de $\mathcal{A}$ est dit $A$-libre s'il est isomorphe à une somme de $A$. Un cofibrant de $s \mathcal{A}$ est dit $A$-libre s'il est $A$-libre en chaque degré. Une cofibration de $s \mathcal{A}$ est dite $A$-libre si sa cofibre est $A$-libre.

Lemme 3.2. Toute cofibration (resp. cofibration appartenant à hB ) de $C h(\mathcal{A})$ est rétracte d'une cofibration $U$-libre (resp. cofibration $U$-libre appartenant à $h \mathcal{B}$ ).

Démonstration. Soit $A \rightarrow B$ une cofibration de cofibre $C$. Comme $U$ est un générateur projectif, on peut toujours choisir des résolutions projectives qui sont sommes de copies de $U$ en chaque degré. Le complexe total d'une résolution de CartanEilenberg [W] pp.145-146] fournit donc une fibration triviale $L \rightarrow C$, avec $L U$ libre. En particulier, si $C$ est $h \mathcal{B}$-acyclique, $L$ l'est également. De plus, comme $C$ est cofibrant, il existe un morphisme $C \rightarrow L$ tel que la composée : $C \rightarrow L \rightarrow C$ soit l'identité. Dans le diagramme commutatif ci-dessous, le carré inférieur droit est cartésien, la flèche $B \rightarrow B^{\prime}$ s'obtenant alors de manière à ce que la composée $B \rightarrow B^{\prime} \rightarrow B$ soit l'identité. L'objet $A^{\prime}$ est le noyau du morphisme $B^{\prime} \rightarrow L$ et les flèches du haut s'obtiennent par restriction.

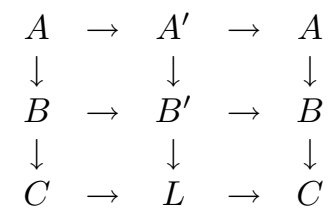

La cofibration $A \rightarrow B$ est alors bien rétracte de la cofibration $U$-libre $A^{\prime} \rightarrow B^{\prime}$, qui appartient à $h \mathcal{B}$ quand il en va de même de $A \rightarrow B$.

Comme $\mathcal{A}$ est une catégorie de Grothendieck, $\mathcal{B}$ est également localisante (proposition A.3 , et le foncteur $T$ préserve générateurs et toute colimite [G, pp.373, 378] [P. pp.180, 186]. Soit $U^{\prime}$ un générateur projectif dans $\mathcal{A} / \mathcal{B}$, et $V=S\left(U^{\prime}\right)$. L'objet $V$ est un projectif de $\mathcal{A}$, et il est $\mathcal{B}$-colocal. 
Proposition 3.3. Soit $V$ le projectif $\mathcal{B}$-colocal défini ci-dessus. Un cofibrant $C$ de $C h(\mathcal{A})$ est $h \mathcal{B}$-acyclique si et seulement s'il existe une équivalence faible : $C^{\prime} \rightarrow C$ dans $C h(\mathcal{A})$, où $C^{\prime}$ est un cofibrant $V$-libre.

Démonstration. D'après le théorème $1.2, C \in C h(\mathcal{A})$ est $h \mathcal{B}$-acyclique si et seulement si $(\boldsymbol{L}(S) \circ \tilde{T})(C) \rightarrow C$ est un isomorphisme dans $H o(C h(\mathcal{A}))$. Il existe $\bar{C}^{\prime} \in \mathcal{A} / \mathcal{B}$ cofibrant $U^{\prime}$-libre, ainsi qu'une équivalence faible $\bar{C}^{\prime} \rightarrow T(C)$, et donc tels que : $\boldsymbol{L}(S)(\tilde{T}(C)) \simeq S\left(\bar{C}^{\prime}\right)$. Posant $C^{\prime}=S\left(\bar{C}^{\prime}\right)$, le morphisme entre cofibrants : $C^{\prime} \simeq(\boldsymbol{L}(S) \circ \tilde{T})(C) \rightarrow C$ de $H o(C h(\mathcal{A}))$ provient donc de $C h(\mathcal{A})$, et $C^{\prime} \rightarrow C$ est une équivalence faible si et seulement si $C$ est $h \mathcal{B}$-acyclique.

3.3. Factorisation. L'objectif est ici de montrer le théorème de factorisation suivant, afin d'achever la démonstration du théorème 3.1.

Théorème 3.4. Soit $f: X \rightarrow Y$ un morphisme de $C h(\mathcal{A})$. Il existe une factori-

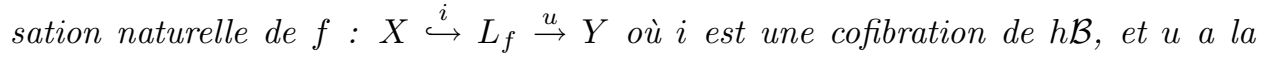
propriété de relèvement à droite par rapport à toute cofibration de $h \mathcal{B}$.

Démonstration. Suivant la méthode de [B1, p.147], la factorisation s'obtient par une version transfinie de "l'argument du petit objet" Q, p.3.4]. Pour cela, il suffit de produire un ensemble de $h \mathcal{B}$-cofibrations triviales dont la source est de taille bornée et représentant la classe de toutes les $h \mathcal{B}$-cofibrations triviales. Ceci est effectué par la proposition 3.8 à la fin de cette section.

On commence par définir la notion de taille utilisée.

Définition. Soit $\beta$ un cardinal. Un objet $A \in \mathcal{A}$ est dit $\beta$-petit s'il est quotient de l'objet libre : $\left(\bigoplus_{J} U\right)$, avec $|J| \leq \beta$. Un complexe $A \in \operatorname{Ch}(\mathcal{A})$ est $\beta$-petit s'il l'est en chaque degré. Un morphisme de $\operatorname{Ch}(\mathcal{A})$ est $\beta$-petit si sa source et son but le sont.

Soient $\alpha$ le plus petit cardinal infini supérieur à $|I|$, et $\gamma$ tel que $V$ soit $\gamma$ petit. Comme $V$ est projectif, il est donc retract de $\left(\bigoplus_{\gamma} U\right)$. Les flèches de $U_{i}$ dans une somme se factorisant par des sous-sommes finies, celles de source $U$ se factorisent par des sous-sommes de cardinal inférieur à $\alpha$, et donc celles de source $V$ se factorisent par des sous-sommes de cardinal inférieur à $\max (\alpha, \gamma)$.

Soit $\rho$ le cardinal $\max (\alpha, \gamma)$.

Soit $\left\{A_{i}\right\}_{i \in I}$ un ensemble d'objets de $C h(\mathcal{A})$. On appelle sous-somme de $\bigoplus_{i \in I} A_{i}$ un objet de la forme $\bigoplus_{i \in J} A_{i}$, où $J$ est un sous-ensemble de $I$.

Lemme 3.5. Tout morphisme $C^{\prime} \stackrel{f}{\rightarrow} C$ avec $C^{\prime} V$-libre et $C U$-libre est colimite filtrante de ses sous-morphismes $\rho$-petit, de source et but sous-somme en chaque degré.

Démonstration. Soit, pour tout $n \in \mathbb{N}, C_{n} \simeq\left(\bigoplus_{i \in I_{n}} U\right)$, un isomorphisme fixé. Soit $A_{n}$ l'ensemble des parties finies de $I_{n}$, et $A=\left(\coprod_{n \in \mathbb{N}} A_{n}\right)$. Soit, pour tout $\alpha \in A$, le sous-complexe de $C$ :

$$
\text { Si } \alpha \in A_{k}: C_{n}^{\alpha}= \begin{cases}0, & n>k, \\ \left(\bigoplus_{\alpha} U\right), & n=k, \\ \text { plus petite sous-somme contenant } \operatorname{Im}\left(d_{k}\right), & n<k,\end{cases}
$$

où $d_{k}: C_{k+1} \rightarrow C_{k}$ désigne la différentielle. Avec ces définitions : $C \simeq \operatorname{Colim}_{\alpha \in A} C^{\alpha}$.

De même, en utilisant des notations similaires, on a : $C^{\prime} \simeq \operatorname{Colim}_{\beta \in B} C^{\prime \beta}$. 
Soit, pour tout $\beta \in B, f^{\beta}=f_{\mid C^{\prime \beta}}: C^{\prime \beta} \rightarrow I C^{\beta}$, où $I C^{\beta}$ désigne le plus petit sous-complexe de $C$ sous-somme en chaque degré contenant $f\left(C^{\prime \beta}\right)$.

Les complexes $C^{\alpha}, C^{\prime \beta}, I C^{\beta}$ ainsi constitués sont $\rho$-petits par construction. Soit, pour tout $\alpha \in A, f^{\alpha}: 0 \rightarrow C^{\alpha}$. Le diagramme de sous-morphismes $\left\{f^{i}\right\}_{i \in(A \cup B)}$ a les propriétés requises.

Soit $d_{\alpha}=\sup \left\{\operatorname{card}\left(\mathrm{E}_{i}(A)\right) / i \in I, A U\right.$-libre ou $V$-libre, $\alpha$-petits $\}$.

On définit par récurrence, pour $k \in \mathbb{N}$ :

- $\alpha_{o}=\rho$,

- $\alpha_{k}=\alpha_{k-1}+\alpha \cdot\left(\rho \cdot \mathrm{d}_{\alpha_{k-1}}\right)$, si $k>0$.

Soit $\mathrm{c}=\sup \left\{\alpha_{k} / k \in \mathbb{N}\right\}$.

Proposition 3.6. Soit $C$ un cofibrant libre non nul de $C h(\mathcal{A})$, hB-acyclique. Il existe un sous-complexe non nul $D$ c-petit, cofibrant libre, $h \mathcal{B}$-acyclique, et en chaque degré sous-somme de $C$.

Démonstration. Pour construire $D$, on considère le diagramme donné par le lemme 3.5 pour l'équivalence faible associée à $C$ par la proposition 3.3 .

Soit un sous-morphisme $f^{\alpha_{o}}: C^{\prime \alpha_{o}} \rightarrow C^{\alpha_{o}}$, tel que : $C^{\alpha_{o}} \neq 0$. Il s'inscrit dans le diagramme commutatif, où, pour tout $i \in I, \mathrm{E}_{i}(f)$ est un isomorphisme :

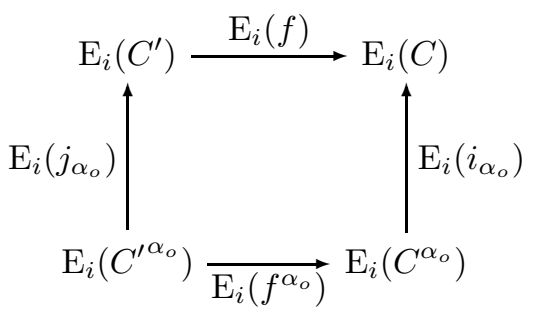

$i_{\alpha_{o}}: C^{\alpha_{o}} \rightarrow C$ (resp. $j_{\alpha_{o}}: C^{\prime \alpha_{o}} \rightarrow C^{\prime}$ ) désignant l'injection canonique.

Pour tout $i \in I$, on a $\mathrm{E}_{i}(f) \simeq \operatorname{Colim}_{\alpha} \mathrm{E}_{i}\left(f^{\alpha}\right)$ où la colimite est prise sur une catégorie filtrante. Pour tout élément $x$ de $\operatorname{Ker}\left(\mathrm{E}_{i}\left(f^{\alpha_{o}}\right)\right),\left(\operatorname{resp} \cdot \operatorname{Coker}\left(\mathrm{E}_{i}\left(f^{\alpha_{o}}\right)\right)\right)$, il existe $\beta_{x}>\alpha_{o}$ tel que $\mathrm{E}_{i}\left(j_{\alpha_{o}}^{\beta_{x}}\right): \mathrm{E}_{i}\left(C^{\prime \alpha_{o}}\right) \longrightarrow \mathrm{E}_{i}\left(C^{\prime \beta_{x}}\right)\left(\operatorname{resp} . \mathrm{E}_{i}\left(i_{\alpha_{o}}^{\beta_{x}}\right)\right)$ envoie $x$ sur 0 .

On définit $f_{1}=\operatorname{Colim}_{j \in J_{1}}\left\{f^{j}\right\}: D_{1}^{\prime} \rightarrow D_{1}$ où $J_{1}$ est l'ensemble des $\beta_{x}$, pour tout $x$ et tout $i \in I$.

De façon similaire, on construit par récurrence, pour $k>0: f_{k}: D_{k}^{\prime} \rightarrow D_{k}$ à partir de $f_{k-1}$. Soit $f_{\infty}=\operatorname{Colim}_{\mathbb{N}} f_{k}$.

Le morphisme $f_{\infty}: D_{\infty}^{\prime} \rightarrow D_{\infty}$ est $c$-petit, de par les constructions de $D_{\infty}^{\prime}$ et $D_{\infty}$ et la définition de $c$. De plus, $f_{\infty}$ est une équivalence faible, car, pour tout $i \in I, \mathrm{E}_{i}\left(f_{\infty}\right)$ est un isomorphisme. Le sous-objet annoncé est $D=D_{\infty}$.

Corollaire 3.7. Soit $A \hookrightarrow B$ une cofibration libre de $h \mathcal{B}$ tel que $B / A \neq 0$. Il existe un objet c-petit $S \in C h(\mathcal{A})$ tel que $S \subset B, S \not \subset A$. De plus, le morphisme $S \cap A \hookrightarrow S$ est une cofibration libre de $h \mathcal{B}$ tel que $S /(S \cap A)$ s'identifie en chaque degré à une sous-somme de $B / A$.

Démonstration. Il suffit d'appliquer la proposition 3.6 à $B / A$.

Proposition 3.8. Soit $f: X \rightarrow Y$ un morphisme de $C h(\mathcal{A})$ ayant la propriété de relèvement à droite par rapport à toutes les cofibrations libres de $h \mathcal{B}$ entre objets c-petits. Alors $f$ a la propriété de relèvement à droite par rapport à toute cofibration de $h \mathcal{B}$. 
Démonstration. C'est essentiellement la démonstration de [B1, p.147].

D'après le lemme 3.2. il suffit de vérifier la propriété de relèvement par rapport aux cofibrations libres de $h \mathcal{B}$. Soit $i: A \hookrightarrow B$ une cofibration libre de $h \mathcal{B}$, et deux flèches $h, k$ faisant commuter le diagramme :

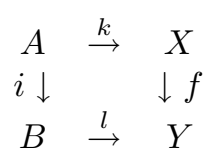

Fixons, pour chaque $q \in \mathbb{N}$, un isomorphisme : $(B / A)_{q} \simeq\left(\bigoplus_{i \in I^{q}} U\right)$. Soit $\mathcal{P}$ l'ensemble des paires $\left(M, k^{M}\right)$ de $\operatorname{Ch}(\mathcal{A})$ telles que :

(i) $A \hookrightarrow M$ et $M \hookrightarrow B$ soient des cofibrations libres de $h \mathcal{B}$,

(ii) Pour tout $q \in \mathbb{N}:(B / A)_{q} \simeq\left(\bigoplus_{i \in I_{M}^{q}} U\right) \stackrel{p_{q}}{\rightarrow}\left(\bigoplus_{i \in I^{q}} U\right) \simeq(B / M)_{q}$, avec $I_{M}^{q} \subset I^{q}$, est la projection canonique,

(iii) le diagramme suivant commute :

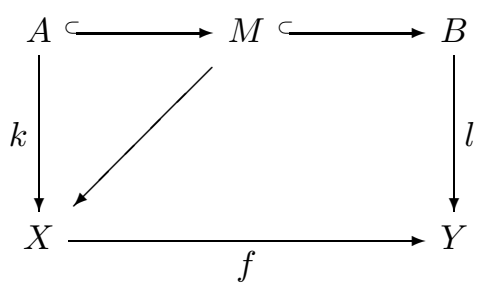

L'ensemble $\mathcal{P}$ est non vide car il contient $(A, k)$.

$\mathcal{P}$ est muni d'une relation d'ordre $:\left(M, k^{M}\right) \geq\left(N, k^{N}\right)$ si $N \subset M\left(I_{N}^{q} \subset I_{M}^{q}\right)$ et $k_{\mid N}^{M}=k^{N}$.

Soit $\mathcal{C}$ une chaîne dans $\mathcal{P}$ et $M_{a j}=\operatorname{Colim}_{\mathcal{C}} M, k_{a j}=\operatorname{Colim}_{\mathcal{C}} k^{M}$.

On a : $\left(B / M_{a j}\right)_{q} \simeq\left(B_{q} / \operatorname{Colim}_{\mathcal{C}} M_{q}\right) \simeq \operatorname{Colim}(B / M)_{q} \simeq\left(\bigoplus_{i \in J q} U\right) \subset(B / A)_{q}$, où $J^{q}=\bigcap_{\mathcal{C}} I_{M}^{q} \subset I^{q}$. Donc $B / M_{a j}$ est cofibrant et $M_{a j} \hookrightarrow B$ une cofibration libre. De plus, $B / M_{a j}$ est $h \mathcal{B}$-acyclique comme colimite $h \mathcal{B}$-acycliques, si bien que $M_{a j} \hookrightarrow B$ est une cofibration libre de $h \mathcal{B}$. C'est donc également le cas de $A \hookrightarrow M_{a j}$. Ainsi, $\left(M_{a j}, k_{a j}\right)$ est un majorant de $\mathcal{C}$. Donc $\mathcal{P}$ possède un élément maximal $\left(M_{a x}, k_{a x}\right)$.

Si $M_{a x} \neq B$, le corollaire 3.7 implique l'existence d'une cofibration libre de $h \mathcal{B}$ : $S \cap M_{a x} \hookrightarrow M_{a x}$, et donc, par hypothèse, d'une flèche : $k_{S}: S \rightarrow X$ faisant commuter le diagramme :

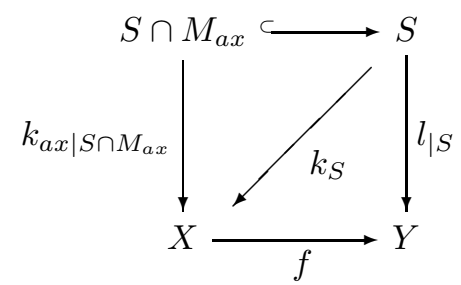

Donc $\left(S \cup M_{a x}, k^{S} \cup k_{a x}\right) \in \mathcal{P}$ et $\left(S \cup M_{a x}, k^{S} \cup k_{a x}\right)>\left(M_{a x}, k_{a x}\right)$. Cette contradiction montre que : $M_{a x}=B$.

\section{Appendix A. Appendice}

A.1. Localisation relativement à une classe de morphisme. Soit $\mathcal{C}$ une catégorie et $\mathcal{W}$ une classe de morphisme de $\mathcal{C}$. 
Définitions. Un objet $L \in \mathcal{C}$ est dit $\mathcal{W}$-local si tout morphisme $X \rightarrow Y$ de $\mathcal{W}$ induit un isomorphisme $\operatorname{Hom}(Y, L) \stackrel{\sim}{\rightarrow} \operatorname{Hom}(X, L)$.

Un objet $C \in \mathcal{C}$ est dit $\mathcal{W}$-colocal si tout morphisme $X \rightarrow Y$ de $\mathcal{W}$ induit un isomorphisme $\operatorname{Hom}(C, X) \stackrel{\sim}{\rightarrow} \operatorname{Hom}(C, Y)$.

Une $\mathcal{W}$-localisation d'un objet $X \in \mathcal{C}$ est la donnée d'un morphisme $X \rightarrow L$ de $\mathcal{W}$ où $L$ est $\mathcal{W}$-local.

Une $\mathcal{W}$-colocalisation d'un objet $X \in \mathcal{C}$ est la donnée d'un morphisme $C \rightarrow X$ de $\mathcal{W}$ où $C$ est $\mathcal{W}$-colocal.

Une monade (resp. comonade) idempotente $(E, \eta)$ est constituée d'un foncteur $E: \mathcal{C} \rightarrow \mathcal{C}$ et d'une transformation naturelle $\eta: I d_{\mathcal{C}} \rightarrow E\left(\right.$ resp. $\eta: E \rightarrow I d_{\mathcal{C}}$ ) tels que $: E \eta=\eta_{E}: E \rightarrow E^{2}$ (resp. $E \eta=\eta_{E}: E^{2} \rightarrow E$ ).

Comme il est indiqué dans [B1, p.134], la donnée d'une $\mathcal{W}$-localisation (resp. $\mathcal{W}$-colocalisation) pour tout objet de $\mathcal{C}$ est équivalente à la donnée d'un foncteur localisation (resp. colocalisation), c'est à dire une monade (resp. comonade) idempotente $(E, \eta)$ telle que, pour tout objet $X$ de $\mathcal{C}: \eta_{X} \in \mathcal{W}$ et $E(X) \mathcal{W}$-local (resp. $\mathcal{W}$-colocal).

De plus, la $\mathcal{W}$-localisation d'un objet $X: X \rightarrow L$ est caractérisée par chacune des propriétés suivantes [B1, p.134] :

- $X \rightarrow L$ est terminal parmi les morphismes appartenant à $\mathcal{W}$ et de source $X$.

- $X \rightarrow L$ est initial parmi les morphismes de but $\mathcal{W}$-local et de source $X$.

Si $\mathcal{C}$ est une catégorie avec un objet nul (i.e. : initial et terminal), noté $*$, on pose également :

Définitions. Un objet $A$ est dit $\mathcal{W}$-acyclique si le morphisme $* \rightarrow A$ appartient à $\mathcal{W}$.

Une $\mathcal{W}$-acyclisation d'un objet $X$ de $\mathcal{C}$ est la donnée d'un $\mathcal{W}$-acyclique $A$ et d'un morphisme $A \rightarrow X$ terminal parmi les morphismes de source $\mathcal{W}$-acyclique et de but $X$.

La donnée d'une $\mathcal{W}$-acyclisation pour tout $X$ de $\mathcal{C}$ est équivalente à la donnée d'un foncteur $\mathcal{W}$-acyclisation, soit un couple $(\mathscr{A}, \theta)$, où $\theta$ est une transformation naturelle : $\mathscr{A} \rightarrow I d$, tel que, pour tout $X$ de $\mathcal{C}, \mathscr{A}(X) \rightarrow X$ soit une $\mathcal{W}$-acyclisation de $X$.

A.2. Localisation et colocalisation dans une catégorie abélienne. Il s'agit de rappels de définitions et résultats ( $[\mathrm{G}, \mathrm{Ch} .3]$ ou $[\mathrm{P}, \mathrm{Ch} .4])$ concernant les situations localisantes et colocalisantes dans une catégorie abélienne.

Dans toute la suite, les catégories abéliennes seront supposées localement petites, i.e. : pour chaque objet, la classe des sous-objets forme un ensemble.

Soient $\mathcal{A}$ une catégorie abélienne, $\mathcal{B}$ une sous-catégorie épaisse de $\mathcal{A}$, c'est-àdire stable par sous-objets, quotients et extensions. On appelle $\mathcal{B}$-isomorphisme les morphismes de $\mathcal{A}$ ayant noyau et conoyau dans $\mathcal{B}$. Il existe une catégorie abélienne $\mathcal{A} / \mathcal{B}$ et un foncteur exact $T: \mathcal{A} \rightarrow \mathcal{A} / \mathcal{B}$ caractérisés par la propriété universelle suivante :

Pour toute catégorie abélienne $\mathcal{C}$ et tout foncteur exact $F: \mathcal{A} \rightarrow \mathcal{C}$ envoyant les $\mathcal{B}$-isomorphismes de $\mathcal{A}$ dans les isomorphismes de $\mathcal{C}$, il existe un unique foncteur $\bar{F}: \mathcal{A} / \mathcal{B} \rightarrow \mathcal{C}$ tel que $: F=\bar{F} \circ T$.

Définition. Soient $\mathcal{A}$ une catégorie abélienne, $\mathcal{B}$ une sous-catégorie épaisse de $\mathcal{A}$. On dit que $\mathcal{B}$ est localisante (resp. colocalisante) si le foncteur passage au quotient : $T: \mathcal{A} \rightarrow \mathcal{A} / \mathcal{B}$ possède un adjoint à droite, noté $R$ (resp. à gauche, noté $S$ ). 
Dans [G, p.371], il est montré que le foncteur $R$ est alors section de $T$, au sens où la co-unité d'adjonction est un isomorphime, si bien que l'unité : $u: I d_{\mathcal{C}} \rightarrow R \circ T$ constitue une monade idempotente. C'est une localisation relative à la classe des $\mathcal{B}$-isomorphismes.

Les propositions suivantes donnent des caractérisations des sous-catégories localisantes. La première est utilisée dans la section 3.

Proposition A.1 ([G p.372]). Soit $\mathcal{B}$ une sous-catégorie épaisse d'une catégorie abélienne $\mathcal{A}$. Les propositions suivantes sont équivalentes:

(i) $\mathcal{B}$ est une sous-catégorie localisante de $\mathcal{A}$.

(ii) Tout objet $A$ de $\mathcal{A}$ contient un sous-objet maximal parmi les sous-objets de $A$ appartenant à $\mathcal{B}$; de plus, si tout sous-objet de $A$ appartenant à $\mathcal{B}$ est nul, il existe un monomorphisme de $A$ dans un objet $\mathcal{B}$-local.

Proposition A.2 ([ㅍ, p.182]). Soit $\mathcal{B}$ une sous-catégorie épaisse d'une catégorie abélienne $\mathcal{A}$ possédant des enveloppes injectives. Les propositions suivantes sont équivalentes:

(i) $\mathcal{B}$ est une sous-catégorie localisante de $\mathcal{A}$.

(ii) Tout objet $A$ de $\mathcal{A}$ possède un sous-objet maximal dans $\mathcal{B}$.

Si, de plus, la catégorie $\mathcal{A}$ possède des sommes quelconques, ces assertions sont équivalentes $[\mathrm{P}, \mathrm{p} .186]$ à:

(iii) $\mathcal{B}$ est stable par somme.

Les énoncés ci-dessus se dualisent, permettant de caractériser les sous-catégories colocalisantes.

Définitions. Un objet $U$ de $\mathcal{A}$ est générateur si le foncteur $\operatorname{Hom}(U,-)$ est pleinement fidèle. Une catégorie abélienne est une catégorie de Grothendieck si elle possède un générateur et des colimites filtrantes exactes.

Exemple : Pour tout anneau $A$, la catégorie des $A$-modules $\mathcal{M o d}_{A}$, et la catégorie de foncteurs $\left(\mathcal{M o d}_{A}\right)^{I}$, où $I$ désigne une petite catégorie quelconque, sont des catégories de Grothendieck.

Proposition A.3 ([NT, p.113]). Soit une catégorie de Grothendieck $\mathcal{A}$ bicomplète. Toute sous-catégorie colocalisante est alors localisante.

Démonstration. Soit $\mathcal{B}$ une sous-catégorie colocalisante de $\mathcal{A}$. Elle est épaisse et stable par produits. Dans une catégorie de Grothendieck, la somme d'un ensemble d'objets est un sous-objet du produit de ces objets. La catégorie $\mathcal{B}$ est donc également stable par sommes.

On a alors affaire à une situation de "recollement" [BBD], également [K, p. 398] :

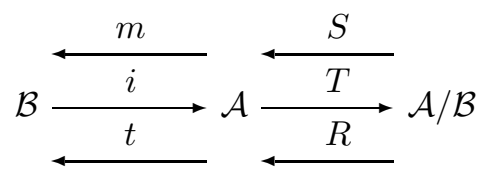

où $S$ (resp. $R$ ) est adjoint à gauche (resp. à droite) du foncteur passage au quotient $T$ et $m$ (resp. $t$ ) est adjoint à gauche (resp. à droite) de l'inclusion $i$. Le foncteur $m$ (resp. $t$ ) est conoyau de la co-unité $: S \circ T \rightarrow I d$ (resp. noyau de l'unité : $I d \rightarrow R \circ T)$, et "plus grand quotient (resp. sous-objet)" appartenant à $\mathcal{B}$. 
A.3. Catégorie de modèle. On rappelle ci-dessous la définition d'une catégorie de modèle fermée de [DS].

Définition ([DS, p.83]). Une catégorie de modèle fermée est une catégorie $\mathcal{C}$ avec trois classes distinguées de morphismes : les équivalences faibles, les fibrations et les cofibrations, chacune fermée pour la composition et contenant les morphismes identités. Un morphisme qui est à la fois une fibration (resp. cofibration) et une équivalence faible est appelée fibration triviale (resp. cofibration triviale). Ces données doivent vérifier les axiomes suivants :

MC1. La catégorie $\mathcal{C}$ possède toute limite finie et toute colimite finie.

MC2. Si $f$ et $g$ sont des morphismes de $\mathcal{C}$ tel que $g f$ est défini et si deux des trois morphismes $f, g$ et $g f$ sont des équivalences faibles, il en est de même de la troisième.

MC3. Si $f$ est un retracte de $g$ et si $g$ est est une fibration, une cofibration, ou une équivalence faible, il en est de même de $f$.

MC4.

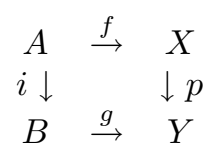

Un relèvement : $B \rightarrow X$ existe dans le diagramme ci-dessus dans chacune des deux situations suivantes : (i) $i$ est une cofibration et $p$ est une fibration triviale, ou (ii) $i$ est une cofibration triviale et $p$ est une fibration.

MC5. Tout morphisme $f$ peut être factorisé de deux manières : (i) $f=p i$, où $i$ est une cofibration et $p$ est une fibration triviale, et (ii) $f=p i$, où $i$ est une cofibration triviale et $p$ est une fibration.

\section{REFERENCES}

[B1] A.K. Bousfield. The localization of spaces with respect to homology. Topology 14, 133-150 (1975) MR 52:1676

[B2] A.K. Bousfield. Constructions of factorization systems in categories. J. Pure Appl. Algebra 9, 207-220 (1977) MR 57:17648

[B3] A.K. Bousfield. The localization of spectra with respect to homology. Topology 18, 257-281 (1979) MR 80m:55006

[BBD] A.A. Beilinson, J. Bernstein, P. Deligne. Faisceaux pervers. Astérisque 100 (1982) MR 86g:32015

[D] A. Dold. Homology of symmetric products and other functors of complexes. Ann. of Math., (2) 68, 54-80 (1958) MR 20:3537

[DP] A. Dold, D. Puppe. Homologie nicht-additiver Funktoren. Anwendungen. Ann. Inst. Fourier 11, 201-312 (1961) MR 27:186

[DHK] W.G. Dwyer, P. Hirshorn, D.M. Kan. Model Categories and More General Abstract Homotopy Theory. http://www-math.mit.edu/ psh/

[DS] W.G. Dwyer, J. Spalinski. Homotopy theories and model categories. James, I. M. (ed.), Handbook of algebraic topology. Amsterdam: North-Holland. 73-126 (1995) MR 96h:55014

[EMcL] S. Eilenberg, S. Mac Lane. On the groups $H(\Pi, n)$. II. Ann. of Math., (2) 60, 49-139 (1954) MR 16:391a

[FS] V. Franjou, J.H. Smith. A duality for polynomial functors. J. Pure Appl. Algebra 104, No.1, 33-39 (1995) MR 96i:18009

[G] P. Gabriel. Des catégories abéliennes. Bull. Soc. Math. France 90, 323-448 (1962) MR 38:1144

[JMcC1] B. Johnson, R. McCarthy. Taylor towers for functors of additve categories. J. Pure Appl. Algebra 137, 253-284 (1999) MR 2000b:18018

[JMcC2] B. Johnson, R. McCarthy. Deriving calculus with cotriples. A paraitre dans Cahiers Topologies Geom. Differentielle Categ. http://www.math.uiuc.edu/ ${ }^{\sim}$ randy/ 
[K] N. Kuhn. Generic representations of the finite general linear groups and the Steenrod algebra. II. K-Theory 8, no. 4, 395-428 (1994) MR 95k:55038

[McL] S. Mac Lane. Categories for the working mathematician. Graduate Texts in Mathematics, 5. New York etc.: Springer-Verlag (1971) MR 50:7275

[MV] F. Morel, V. Voevodsky. $A^{1}$-homotopy theory of schemes, Inst. Hautes Études Sci. Math. No. 90, 45-143 (1999) CMP 2001:08

[NT] C. Nastasescu, B. Torrecillas. Colocalization on Grothendieck categories with applications to coalgebras. J. Algebra 185, No.1, 108-124 (1996) MR 98g:18011

[Pi] T. Pirashvili. Higher additivizations. Trudy Tbiliss. Mat. Inst. Razmadze Akad. Nauk Gruzin. SSR 91, 44-54 (1988) MR 90k:18012

[P] N. Popescu. Abelian categories with applications to rings and modules. L.M.S. Monographs. 3. London-New York: Academic Press. XII (1973) MR 49:5130

[Q] D.G. Quillen. Homotopical Algebra. Lecture Notes in Mathematics. 43. Berlin-HeidelbergNew York: Springer-Verlag (1967) MR 36:6480

[R] B. Richter. Taylor towers for abelian $\Gamma$-groups. Prépublication, Université de Bonn (1999)

[W] C.A. Weibel. An introduction to homological algebra. Cambridge Studies in Advanced Mathematics. 38. Cambridge: Cambridge University Press (1994) MR 95f:18001

Department of Mathematics, Aarhus University, 8000 Aarhus C, Denmark

E-mail address: renaudin@imf.au.dk 